\title{
PERSPECTIVES
}

\section{Anaesthetic preconditioning and its role in protecting patients at risk of myocardial ischaemia}

\author{
Alastair Duggie and Jacob McDermott \\ Department of Anaesthesia, University Hospital of North Durham, Durham, United Kingdom
}

Abstract

Ischaemic preconditioning is a phenomenon where prior minor ischaemic events allows organs to better withstand further episodes of ischaemia. Preconditioning downgrades the effects of ischaemia from necrosis to apoptosis to cell survival. It occurs in a wide variety of tissues, but it is most widely studied in the heart, and it occurs after a range of stimuli including hypoxia and the use of volatile anaesthetic agents. In this article, we look at the basic science, mechanisms, and potential uses of preconditioning.

Keywords: Anaesthetic volatile agents, ischaemic preconditioning, cardiac surgery, anaesthetic preconditioning

Received: 29 October; Accepted: 30 November 2021; Published: 3 I December 2021

\section{Introduction}

There is growing evidence that volatile agents may offer organs protection from ischaemia, in particular, the heart. Volatile agents appear to offer both short- and long-term protection from ischaemic insults [1], which is known as anaesthetic preconditioning. This article describes the types of preconditioning and the mechanisms by which they are thought to occur. It also describes the clinical application of preconditioning, focussing on its role in cardiac protection.

Preconditioning occurs across a wide variety of tissues; however, it was first noted to prevent ischaemia in the myocardium. The heart is one of the few organs where it is possible to measure function or damage (troponins, echocardiograms, etc.), meaning it has been the most widely investigated. Later preconditioning has also been observed in the brain, and now, it has been suggested to offer protection to the liver, kidneys, muscle and gut.

\section{Types of preconditioning}

\section{Ischaemic preconditioning}

After a period of minor ischaemia, organs undergo biomolecular changes that allow them to better withstand further episodes of ischaemia; this is called ischaemic preconditioning [2]. Ischaemic preconditioning was the first type of preconditioning to be discovered and is consequently the most studied.

Remote preconditioning is related to ischaemic preconditioning. In remote preconditioning, distant myocardium is protected from ischaemia following an area of myocardium being exposed to sustained ischaemia [3]. This suggests that preconditioning may be caused by substances produced in one area of the heart and transported to a distant area. Remote preconditioning has also been demonstrated using a tourniquet on an upper limb [4].

\section{Anaesthetic preconditioning}

All modern volatile agents (halothane, enflurane, isoflurane, desflurane and sevoflurane) appear to cause preconditioning [5]. From animal studies, it appears that desflurane has the greatest protective effect of all the volatile agents, followed by sevoflurane, and then isoflurane [6]. It also appears that other drugs used in anaesthesia, including morphine and lidocaine, have preconditioning properties $[7,8]$

\section{Evidence}

Most of the evidence for all types of preconditioning is obtained from animal studies. It is difficult to evaluate in humans due to obvious difficulty in studying an organ after an ischaemic event; clearly damaging human organs purely for research is unethical. The availability of markers of myocardial damage in humans makes the heart the most studied organ. As ischaemia to an organ is an unpredictable event, most of these studies have taken place during coronary artery bypass grafting $(\mathrm{CABG})$ or during percutaneous angioplasty (PCA), where periods of ischaemia can be predicted. 


\section{Laboratory}

There is a wide range of evidence showing preconditioning in laboratory studies. Dogs that have been given isoflurane prior to the left anterior descending artery being occluded showed better recovery of myocardial function [9]. This was initially thought to be due to vasodilation of the coronary arteries or collateral circulation or decreased metabolic rate caused by the volatile gas. However, if the isoflurane had been washed out for 5 or $30 \mathrm{~min}$, the myocardial function was still preserved [10]. This suggests that the anaesthetic gases brought about a change, which protected the myocardium. In another study, human right atrial muscles taken during $\mathrm{CABG}$, which were exposed to desflurane for $15 \mathrm{~min}$, showed an improved contractile recovery from a prolonged period of ischaemia [11]. This has also been repeated in tissues, which were treated with opioid, again with similar results [12]. In addition, lidocaine infusion has been shown to protect isolated rat hearts from ischaemia [8].

\section{Ischaemic preconditioning}

Ischaemic preconditioning, in addition to its laboratory evidence, has some evidence in humans in relation to 'walk through angina'. 'Walk through angina' is where someone has increased exercise tolerance a few minutes after experiencing angina due to exertion. It has been observed that patients who have episodes of angina just prior to having a myocardial infarction (MI) have a better prognosis from the MI [13]. The ischaemic preconditioning effect has been prevented by administering adenosine antagonists [14], opioid receptor antagonists [15] and K-ATP channel blockers [16]; suggesting that these have a role to play in preconditioning.

\section{Anaesthetic preconditioning}

Direct evidence for anaesthetic preconditioning is difficult to produce; however, there are some limited studies, most of which involve CABG. In one of the studies, an isoflurane anaesthetic was shown to lead to a decrease in troponin levels postoperatively [17]. In another study, the use of enflurane leads to better post-ischaemic function despite levels of troponin and creatine kinase $\mathrm{C}$ being unchanged [18]. It should be noted, however, that the numbers of subjects in these groups are small, that is, around 20-30 subjects in each trial, and there are many confounding factors that could influence these results.

\section{Clinical trials}

Clinical trials into anaesthetic preconditioning are difficult to conduct, so few exist. The Remote Ischemic Preconditioning for Heart Surgery (RIPHeart) study was a large multicentre study, which examined whether remote ischaemic preconditioning (using a tourniquet on the participant's leg) would have any effect on mortality, morbidity or cardiac function after major cardiac surgery [19]. The study found that remote ischaemic preconditioning conferred no clinical improvement and had no effect on cardiac function on transoesophageal echocardiography immediately post-surgery, and after 1 year.

A Cochrane meta-analysis comparing volatile agents with total intravenous anaesthetic (TIVA) for patients undergoing cardiac surgery showed significant improvement in mortality from use of volatile agents [20]. However, the meta-analysis had several limitations; many of the trials included were small and not double-blinded, the indirect evidence used in the Bayesian analysis is subject to confounding, and once the largest study was removed, only desflurane was shown to confer any benefit. However, with increasing focus on the environmental effects of volatile agents, this will undoubtedly generate an interesting discussion.

\section{Mechanisms}

It is likely that volatile agents cause preconditioning via several mechanisms. They act at receptors via a second messenger system (most likely protein kinase C) on receptors in the mitochondria or alter the production of reactive oxygen species (ROS). They cause the transcription of new proteins, which may lead to the late phase of preconditioning. To fully understand the mechanism of action, we consider ischaemia and the role of apoptosis and necrosis.

\section{Ischaemia}

During periods of ischaemia, there is a decrease in adenosine triphosphate (ATP) production, leading to the $\mathrm{Na} / \mathrm{K}$ pumps being inhibited and a consequent build-up of $\mathrm{Na}^{+}$in the cell. As a result, there is also an accumulation of $\mathrm{H}^{+}$ions within the cell. To preserve $\mathrm{pH}$ levels, hydrogen ions are excreted from the cell in exchange for $\mathrm{Na}^{+}$and $\mathrm{Ca}^{2+}$, and some of the $\mathrm{Na}^{+}$is exchanged for $\mathrm{Ca}^{2+}$. High levels of $\mathrm{Ca}^{2+}$ ions within the cell lead to apoptosis, and at higher levels, necrosis.

\section{Apoptosis}

In response to ischaemia, a cell can either survive, undergo apoptosis or undergo necrosis, with worsening effects for the organ. Preconditioning downgrades the reaction from necrosis to apoptosis or cell survival. Apoptosis is the programmed destruction of a cell; this is energy dependent and is tightly regulated. During apoptosis, the contents of the cell are broken down in a controlled manner, with the cell wall broken down last, meaning that the antigenic material is not released. There is no inflammatory response, and further damage to the organ is minimised. If a cell undergoes necrosis, the cell bursts and antigenic material is released, leading to an inflammatory reaction. Consequently, there is further organ damage. Apoptosis is mediated by mitochondria. 


\section{Adenosine}

Receptors have an important role in preconditioning. There are multiple receptors involved, having varying degrees of importance. In ischaemic preconditioning, it has been shown that the release of adenosine and its effects on the A1 receptor are particularly significant. Ischaemia of the myocardium leads to the release of adenosine, which has several protective effects [21]. These include vessel vasodilatation, which increases oxygen delivery to the heart, reduced chronotropy and inotropy, leading to reduced oxygen demand, decreased free radical release causing reduced vascular injury and inhibition of platelet aggregation. Volatile anaesthetic agents are thought to activate adenosine A1 receptors, mimicking these effects [9].

In addition, it has been shown that preconditioning effects can be reduced by blocking adenosine receptors [14]. 8-Cyclopentyl-1,3-dipropylxanthine is an A1 receptor antagonist; however, it has been shown to only partially reduce the preconditioning effects of isoflurane [22]. This would suggest that isoflurane acts on other receptors to cause preconditioning.

\section{Second messengers}

Protein kinase are a group of second messenger proteins that are implicated in anaesthetic preconditioning [22, 23]. Sevoflurane and isoflurane both cause protein kinase $\mathrm{C}$ to transfer to cytosol within the cell. Protein kinase $\mathrm{C}$ has effects on the ATP-dependent $\mathrm{K}^{+}$channels and regulates the production of ROS. Protein kinase B second messengers that inhibit apoptosis have been shown to be activated by anaesthetic volatile agents [24]. Cardiac protective effects of ischaemic preconditioning were found to be completely eliminated by infiltration of the combination of tyrosine kinase and protein kinase inhibitors [25]

Nitric oxide is known to be involved in preconditioning. Preconditioning effects caused by desflurane in animal studies have been shown to be reversed by nitric oxide inhibitors; however, the exact role of nitric oxide is unclear [26].

In animal studies, injection of a PI3K inhibitor was shown to lead to increased cardiac damage after an ischaemic insult [27]. PI3K is implicated in the reperfusion injury salvage kinase (RISK) pathway, which is found to play a major role in preconditioning.

Finally, bradykinin is also thought to play a role in preconditioning. Activation of bradykinin receptors in rats by exogenous administration of bradykinin has shown to cause preconditioning; however, the mechanism is unclear [28].

\section{ATP K-dependent channels}

ATP K-dependent channels in the mitochondria are of vital importance in preconditioning [10]. When these open there is an influx of $\mathrm{K}$ into the inner membrane of
Table 1. Summary of mechanisms of preconditioning

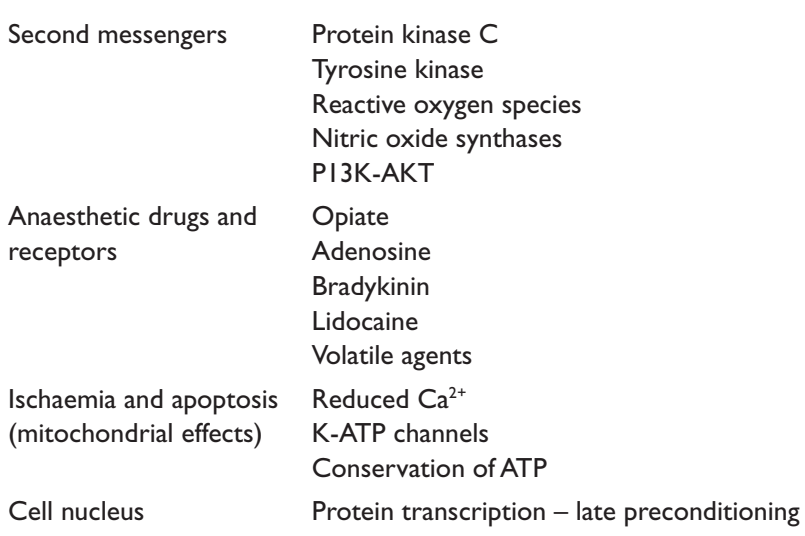

the mitochondria, causing depolarisation of the mitochondria and a disruption of ATP production [10]. This limits the swelling of mitochondria by closure of other ion channels, and importantly limits the amount of $\mathrm{Ca}^{2+}$ ions entering the mitochondria. High levels of $\mathrm{Ca}^{2+}$ lead to apoptosis or necrosis of the mitochondria with consequent damage to the cell and organs. Volatile agents directly open these channels, and drugs that block these channels prevent preconditioning.

\section{Reactive oxygen species}

As part of their normal function, the mitochondria produce a small amount of ROS, which include hydrogen peroxide (H2O2-) and superoxide (O2-). These react with proteins and cause damage. This is an important function in human physiology as they can remove damaged or unwanted proteins; however, at high levels this can become unregulated with resulting damage to the cell. It has been shown that volatile agents decrease dityrosine formation (a marker of ROS) and protect from this effect [29].

\section{Uses of preconditioning}

The potential uses of anaesthetic preconditioning are extensive. The first obvious group is in the high-risk surgical patient, particularly patients undergoing major surgery. One of the major causes of death in this group is from co-existing cardiovascular conditions. Anaesthetic preconditioning has the potential to reduce this by limiting the effect of ischaemia. Clinical trials looking into effects of anaesthetic preconditioning have so far been inconclusive. The low incidence of clinically relevant endpoints, such as mortality, makes this a difficult area to investigate. So far, uses for anaesthetic preconditioning have been theoretical.

\section{Coronary artery bypass grafting}

With coronary bypass grafting there is considerable mortality from damage to the myocardium for patients with 
pre-existing cardiac disease [30]. There is also the chance of a stroke during bypass. Anaesthesia is maintained during bypass by infusion of propofol or injection of a volatile agent from the bypass circuit. As discussed above, volatile agents may offer cardiac protection and a faster recovery, as suggested by lower post-operative troponin levels [17].

During an angiogram, there is some interruption of blood supply to the organ being imaged. While most of this work is undertaken under sedation, it may be that those patients with high risk of complications may be better served by carrying this out under volatile anaesthetic. This may also be the case in other procedures in high-risk patients, which are usually carried out under sedation.

\section{Controversies}

The clinical implications of anaesthetic preconditioning are still unclear. The most likely group to benefit would seem to be the high-risk surgical group, those undergoing cardiac surgery and those with pre-existing cardiac conditions; however, there may be other groups that could also benefit. Traditionally, high-risk surgical groups have significant respiratory or cardiovascular co-morbidities, so these may already have ischaemic preconditioning, and it may be that anaesthetic preconditioning offers no additional benefit.

There are many uncertainties surrounding anaesthetic preconditioning. It is not clear how much exposure to volatile agents is required for preconditioning to occur in humans, and if there is a dose-dependent benefit. Also, the length of time of exposure required for preconditioning to occur has not been well demonstrated. There is the possibility that other drugs may confer these benefits.

Most of the currently available evidence comes from animal studies or relates to other forms of preconditioning, such as remote preconditioning. Generating clinical evidence of the benefit of anaesthetic preconditioning is extremely difficult. To conduct a large, well-designed trial to show direct clinical outcomes from the use of anaesthetic preconditioning would be fraught with difficulty. There are many confounding factors affecting clinical endpoints, so it is likely that a sufficiently powered trial would have been very large. It would be unethical to conduct a double-blind trial into this subject, as it could potentially affect patient safety.

\section{Conclusion}

There is little doubt of the existence of anaesthetic preconditioning as a phenomenon within a wide variety of tissues. However, its clinical relevance has still to be established. It is difficult to study the effects or magnitude in clinical situations. Part of this issue arises from the variety of ways from which preconditioning occurs.
Clinical trials would be either unethical, unsafe or have large amounts of confounding factors, which could influence the results. Despite this, there are some small clinical trials, suggesting that volatile agents may have some benefits to the myocardium. It will take further work to either confirm or refute this. However, there is the potential for an exciting change in the way that volatile agents are used, or for there to be a new category of patients who could benefit from an anaesthetic.

\section{Conflict of interest and funding}

The authors have not received any funding or benefits from the industry or elsewhere to conduct this study.

\section{References}

1. Yellon D, Downley J. Preconditioning the myocardium: from cellular physiology to clinical cardiology. Physiol Rev 2003; 83: 1113-51. doi: 10.1152/physrev.00009.2003

2. Murray C, Jennings R, Reimer K. Preconditioning with ischemia: a delay of lethal cell injury in ischemic myocardium. Circulation1986; 74:1124-36. doi: 10.1161/01.CIR.74.5.1124

3. Przyklenk K, Bauer B, Ovize M, Kloner RA, Whittaker, P. Regional ischemic 'preconditioning' protects remote virgin myocardium from subsequent sustained coronary occlusion. Circulation 1993; 87: 893-9. doi: 10.1161/01.CIR.87.3.893

4. Lai I, Chang K, Chen C, Tsai H. Transient limb ischemia induces remote preconditioning in liver among rats: the protective role of heme oxygenase-1. Transplantation 2006; 81: 131117. doi: $10.1097 / 01 . t p .0000203555 .14546 .63$

5. Kunst G, Klein A. Peri-operative anaesthetic myocardial preconditioning and protection-cellular mechanisms and clinical relevance in cardiac anaesthesia. Anaesthesia 2015; 70: 467-82. doi: 10.1111/anae. 12975

6. Redel A, Stumpner J, Tischer-Zeitz T, Lange M, Smul T, Lotz C, et al. Comparison of isoflurane-, sevoflurane-, and desflurane-induced pre-and postconditioning against myocardial infarction in mice in vivo. Exp Biol Med. 2009;234: 1186-91. doi: 10.3181/0902-RM-58

7. Schultz J, Gross G. Opioids and cardioprotection. Pharmacol Therap 2001; 89: 123-37. doi: 10.1016/S0163-7258(00)00106-6

8. Ebel D, Lipfert P, Frässdorf J, Preckel B, Müllenheim J, Thämer $\mathrm{V}$, et al. Lidocaine reduces ischaemic but not reperfusion injury in isolated rat heart. $\mathrm{Br} \mathrm{J}$ Anaesth 2001; 86(6): 846-52. doi: $10.1093 / \mathrm{bja} / 86.6 .846$

9. Davis R, Sidi A. Effect of isoflurane on the extent of myocardial necrosis and on systemic hemodynamics, regional myocardial blood flow, and regional myocardial metabolism in dogs after coronary artery occlusion. Anesth Analg 1989; 69. doi: 10.1213/ 00000539-198911000-00005

10. Kersten J, Schmeling T, Pagel P, Gross G, Warltier D. Isoflurane mimics ischemic preconditioning via activation of KATPChannels: reduction of myocardial infarct size with an acute memory phase. J Am Soc Anesthesiol 1997; 87: 361-70. doi: 10.1097/00000542-199708000-00024

11. Hanouz J, Yvon A, Massetti M, Lepage O, Babatasi G, Khayat A, et al. . Mechanisms of desflurane-induced preconditioning in isolated human right atria in vitro. Anesthesiol J Am Soc Anesthesiol 2002; 97: 33-41. doi: 10.1097/00000542-199708000-00024

12. Bell S, Sack M, Patel A, Opie L, Yellon D. Delta opioid receptor stimulation mimics ischemic preconditioning in human heart 
muscle. J Am Col Cardiol 2000; 36: 2296-302. doi: 10.1016/ S0735-1097(00)01011-1

13. Solomon S, Anavekar N, Greaves S, Rouleau J, Hennekens C. Angina pectoris prior to myocardial infarction protects against subsequent left ventricular remodeling. J Am Col Cardiol. 2004; 43: 1511-14. doi: 10.1016/j.jacc.2003.09.069

14. Armstrong S, Downey J, Ganote C. Preconditioning of isolated rabbit cardiomyocytes: induction by metabolic stress and blockade by the adenosine antagonist SPT and calphostin $\mathrm{C}$, a protein kinase C inhibitor. Cardiovasc Res 1994; 28: 72-7. doi: 10.1093/cvr/28.1.72

15. Schultz J, Rose E, Yao Z, Gross G. Evidence for involvement of opioid receptors in ischemic preconditioning in rat hearts. Am J Physiol Heart Circu Physiol 1995; 268: 2157-61. doi: 10.1152/ ajpheart.1995.268.5.H2157

16.Fryer R, Eells J, Hsu A, Henry M, Gross G. Ischemic preconditioning in rats: role of mitochondrial K-ATP channel in preservation of mitochondrial function. Am J Physiol-Heart Circu Physiol2000;278:305-12.doi: 10.1152/ajpheart.2000.278.1.H305

17. Tomai F, De Paulis R, Penta D, Colagrande L, Caprara E, Polisca P, et al. Beneficial impact of isoflurane during coronary bypass surgery on troponin I release. Giornale italiano di cardiologia 1999; 29: 1007.

18. De Peppo APPPF, De Paulis R, Turani F, Zupancich E, Sommariva L, Pasqualetti P, et al. Recovery of LV contractility in man is enhanced by preischemic administration of enflurane. Ann Thorac Surg 1999; 68: 112. doi: 10.1016/S0003-4975(99)00466-X

19. Meybohm P, Kohlhaas M, Stoppe C, Gruenewald M, Renner $\mathrm{J}$, Bein B, et al. RIPHeart (remote ischemic preconditioning for heart surgery) study: myocardial dysfunction, postoperative neurocognitive dysfunction, and 1 year follow-up. J Am Heart Assoc 2018; 7(7): e008077. doi: 10.1161/JAHA.117.008077

20. Landoni G, Greco T, Biondi-Zoccai G, Nigro Neto C, Febres D, Pintaudi M, et al. Anaesthetic drugs and survival: a Bayesian network meta-analysis of randomized trials in cardiac surgery. Br J Anaesth 2013; 111(6): 886-96. doi: 10.1093/bja/aet231

21. Liu G, Thornton J, Van Winkle D, Stanley A, Olsson R, DowneyJ. Protection against infarction afforded by preconditioning is mediated by A1 adenosine receptors in rabbit heart. Circulation 1991; 84: 350-6. doi: 10.1161/01.CIR.84.1.350

22. Zheng S Zuo Z. Isoflurane preconditioning decreases glutamate receptor overactivation-induced Purkinje neuronal injury in rat cerebellar slices. Brain Res 2005; 1054: 143-51. doi: 10.1016/j. brainres.2005.06.064

23. Ytrehus K, Liu Y, Downey J. Preconditioning protects ischemic rabbit heart by protein kinase $\mathrm{C}$ activation. J Physiol-Heart Circu Physiol. 1994; 266: 1145-52. doi: 10.1152/ajpheart.1994. 266.3.H1145

24. Feng J, Fischer G, Lucchinetti E, Zhu M, Bestmann L, Jegger D. Infarct-remodeled myocardium is receptive to protection by isoflurane postconditioning role of protein kinase B/Akt signaling. Anesthesiol J Am Soc Anesthesiol 2006; 104(5): 1004-14. doi: 10.1097/00000542-200605000-00017

25. Vahlhaus C, Schulz R, Post H, Rose J, Heusch G. Prevention of ischemic preconditioning only by combined inhibition of protein kinase $\mathrm{C}$ and protein tyrosine kinase in pigs. J Mol Cell Cardiol 1998; 1(30): 197-208. doi: 10.1006/jmcc.1997.0609

26. Smul TM, Lange M, Redel A, Burkhard N, Roewer N, Kehl F. Desflurane-induced preconditioning against myocardial infarction is mediated by nitric oxide. J Am Soc Anesthesiol 2006; 105(4): 719-25. doi: 10.1097/00000542-200610000-00018

27. Rossello X, Riquelme J, Davidson S, Yellon D. Role of PI3K in myocardial ischaemic preconditioning: mapping pro-survival cascades at the trigger phase and at reperfusion. J Cell Mol Med 2018; 22(2): 926-35.

28. Schoemaker R, van Heijningen C. Bradykinin mediates cardiac preconditioning at a distance. Am J Physiol-Heart Circu Physiol. 2000; 278(5): 1571-6. doi: 10.1152/ajpheart.2000.278.5.H1571

29. Novalija E, Varadarajan S, Camara A, An J, Chen Q, Riess M, et al. Anesthetic preconditioning: triggering role of reactive oxygen and nitrogen species in isolated hearts. Am J Physiol - Heart Circu Physiol 2002; 283:44-52. doi: 10.1152/ajpheart.01056.2001

30. Bounous E, Mark D, Pollock B, Hlatky M, Harrell FJ, Lee K, et al. Surgical survival benefits for coronary disease patients with left ventricular dysfunction. Circulation 1988; 78: 1151-7.

\footnotetext{
*Alastair Duggie

Department of Anaesthetics

University Hospital Of Durham

North Road DURHAM DHI 5TW

United Kingdom

Email: a.duggie@nhs.net
} 